\title{
How to Sign Digital Streams
}

\author{
Rosario Gennaro and Pankaj Rohatgi \\ I.B.M. T.J.Watson Research Center \\ P.O.Box 704, Yorktown Heights, NY 10598, U.S.A. \\ Email: rosario, rohatgi@watson.ibm.com
}

\begin{abstract}
We present a new efficient paradigm for signing digital streams. The problem of signing digital streams to prove their authenticity is substantially different from the problem of signing regular messages. Traditional signature schemes are message oriented and require the receiver to process the entire message before being able to authenticate its signature. However, a stream is a potentially very long (or infinite) sequence of bits that the sender sends to the receiver and the receiver is required to consumes the received bits at more or less the input rate and without excessive delay. Therefore it is infeasible for the receiver to obtain the entire stream before authenticating and consuming it. Examples of streams include digitized video and audio files, data feeds and applets. We present two solutions to the problem of authenticating digital streams. The first one is for the case of a finite stream which is entirely known to the sender (say a movie). We use this constraint to devise an extremely efficient solution. The second case is for a (potentially infinite) stream which is not known in advance to the sender (for example a live broadcast). We present proofs of security of our constructions. Our techniques also have applications in other areas, for example, efficient authentication of long files when communication is at a cost and signature based filtering at a proxy server.
\end{abstract}

\section{Introduction}

Digital Signatures (see $[5,17])$ are the cryptographic answer to the problem of information authenticity. When a recipient receives digitally signed information and she is able to verify the digital signature then she can be certain that the information she received is exactly the same as what the sender (identified by his public key) has signed. Moreover, this guarantee is non-repudiable, i.e., the entity identified by the public key cannot later deny having signed the information. Thus, the recipient can hold the signer responsible for the content she receives. ${ }^{2}$ However current digital signature technology was designed to ensure message authentication and its straightforward application does not yield a satisfactory

\footnotetext{
1 This distinguishes digital signatures from message authentication codes (MAC) which allow the receiver to have confidence on the identity of the sender, but not to prove to someone else this fact, i.e. MAC's are repudiable.
} 
solution when applied to information resources which are not message-like. In this paper we discuss one such type of resource: streams. We point out shortcomings in several approaches (some of them used in practice) to tackle the problem of signing streams and then present our solution which does not have such shortcomings.

\subsection{Streams Defined}

A stream is a potentially very long (infinite) sequence of bits that the sender sends to the receiver. The stream is usually sent at a rate which is negotiated between the sender and receiver or there may be a demand-response protocol in which the receiver repeatedly sends requests for additional (finite) amount of data. The main features of streams which distinguish them from messages is that the receiver must consume the data it receives at more or less the input rate, i.e., it can't buffer large amounts of unconsumed data. In fact in many applications the receiver stores relatively very small amounts of the stream. In some cases the sender itself may not store the entire sequence, i.e., it may not store the information it has already sent out and it may not know anything about the stream much beyond what it has sent out.

There are many examples of digital streams. Common examples include digitized video and audio which is now routinely transported over the Internet and also to television viewers via various means, e.g., via direct broadcast satellites and very shortly via cable, wireless cable, telephone lines etc. This includes both prerecorded and stored audio/video programming as well as live feeds. Apart from audio/video, there are also data feeds (e.g., news feeds, stock market quotes etc) which are best modeled as a stream. The Internet and the emerging interactive TV industry also provides another example of an information resource which is best modeled as a stream, i.e., applets. Most non-trivial applets are actually very large programs which are organized into several modules. The consumer's machine first downloads and executes the startup module and as the program proceeds, additional modules are downloaded and executed. Also, modules which are no longer in use may be discarded by the consumer machine. This structure of applets is forced by two factors. Firstly the amount of storage available on the consumer machine may be limited (e.g., in the emerging interactive TV industry set-top boxes have to be cheap and therefore resource limited). Secondly (in the case of the Internet), the bandwidth available to download code may be limited and applets must be designed to start executing as soon as possible. Also it is quite likely that some of the more sophisticated applets may have data-rich components generated on the fly by the applet server. Therefore applets fit very nicely into the demand/response streams paradigm.

Given the above description, it is clear that message oriented signature schemes cannot be directly used to sign streams since the receiver cannot be expected to receive the entire stream before verifying the signature. If a stream is infinitely 
long (e.g., the 24-hours news channel), then it is impossible for the receiver to receive the entire stream and even if a stream is finite but long the receiver would have to violate the constraint that the stream needs to be consumed at roughly the input rate and without delay.

\subsection{Previous Solutions and their Shortcomings}

Up to the authors' knowledge there has been no proposed specific solution to the problem of signing digital streams in the crypto literature. One can envision several possible solutions, some of them are actually proposed to be used in practice.

One type of solution splits the stream in blocks. The sender signs each individual block and the receiver loads an entire block and verifies its signature before consuming it. This solution also works if the stream is infinite. However this solution forces the sender to generate a signature for each block of the stream and the receiver to verify a signature for each block. With today's signature schemes either one or both of these operations can be very expensive computationally. Which in turns means that the operations of signing and verifying can create a bottleneck to the transmission rate of the stream.

Another type of solution works for only finite streams. In this case, once again the stream is split into blocks. Instead of signing each block, the sender creates a table listing cryptographic hashes of each of the blocks and signs this table. When the receiver asks for the authenticated stream, the sender first sends the signed table followed by the stream. The receiver first receives and stores this table and verifies the signature on it. If the signature matches then the receiver has the authenticated cryptographic hash of each of blocks in the stream and thus each block can be verified when it arrives. The problem with this solution is that it requires the storage and maintenance of a potentially very large table on the receiver's end. In many realistic scenarios the receiver buffer is very limited compared to the size of the stream, (e.g., in MPEG a typical movie may be 20 GBytes whereas the receiver buffer is only required to be around $250 \mathrm{Kbytes}$ ). Therefore the hash table can itself become fairly large (e.g., 50000 entries in this case or $800 \mathrm{Kbytes}$ for the MD5 hash function) and it may not be possible to store the hash table itself. Also, the hash table itself needs to be transmitted first and if it is too large then there will be a significant delay before the first piece of the stream is received and consumed. To address the problem of large tables one can also come up with a hybrid scheme in which the stream is split in consecutive pieces and each piece is preceded by a small signed table of contents. ${ }^{2}$

${ }^{2}$ This is the case now (Java Developer Kit 1.1) for large signed java applets which are distributed as a collection of Java archives (JAR) where each archive has a signed table of hashes of contents and the archives are loaded in the order given in the HTML page in which the applet is embedded. 
The above solution can be further modified by using an authentication tree: the blocks are placed as the leaves of a binary tree and each internal node takes as a value the hash of its children (see [13].) This way the sender needs to sign and send only the root of this tree. However in order to authenticate each following block the sender has to send the whole authentication path (i.e. the nodes on the path from the root to the block, plus their siblings) to the receiver. This means that if the stream has $k$ blocks, the authentication information associated with each block will be $O(\log k)$.

As we will see briefly our solution eliminates all these shortcomings. The basic idea works for both infinite and finite streams, only one expensive digital signature is ever computed, there are no big tables to store, and the size of the authentication information associated with each block does not depend on the size of the stream.

NON-REPUDIATION. Notice that if the receiver were only interested in establishing the identity of the sender, a solution based on MAC would suffice. Indeed once the sender and receiver share a secret key, the stream could be authenticated block by block using a MAC computation on it. Since MAC's are usually faster than signatures to compute and verify, this solution would not incur the computational cost associated with the similar signature-based solution described above.

However a MAC-based approach would not enjoy the non-repudiation property. We stress that we require such property for our solution. Also in order for this property to be meaningful in the context of streams we need to require that each prefix of the stream to be non-repudiable. That is, if the stream is $B=B_{1}, B_{2}, \ldots$ where each $B_{i}$ is a block, we require that each prefix $B_{i}=B_{1} \ldots B_{i}$ is nonrepudiable. This rules out a solution in which the sender just attaches a MAC to each block and then signs the whole stream at the end.

This is to prevent the sender from interrupting the transmission of the stream before the non-repudiability property is achieved. Also it is a guarantee for the receiver. Consider indeed the following scenario: the receiver notices that the applets she is downloading are producing damages to her machine. She interrupts the transfer in order to limit the damage, but at the same time she still wants some proof to bring to court that the substream downloaded so far did indeed come from the sender.

\subsection{Our solution in a nutshell}

Our solution makes some reasonable/practical assumptions about the nature of the streams being authenticated.

First of all we assume that it is possible for the sender to embed authentication information in the stream. This is usually the case, see Section 7 to see how to do this in most real-world situations like MPEG video/audio. We also assume that the receiver has a "small" buffer in which it can first authenticate the received bits 
before consuming them. Finally we assume that the receiver has processing power or hardware that can compute a small number of fast cryptographic checksums faster than the incoming stream rate while still being able to play the stream in real-time.

The basic idea of our solution is to divide the stream into blocks and embed some authentication information in the stream itself. The authentication information of the $i^{\text {th }}$ block will be used to authenticate the $(i+1)^{\text {st }}$ block. This way the signer needs to sign just the first block and then the properties of this single signature will "propagate" to the rest of the stream through the authentication information. Of course the key problem is to perform the authentication of the internal blocks fast. We distinguish two cases.

In the first scenario the stream is finite and is known in its entirety to the signer in advance. This is not a very limiting requirement since it covers most of the Internet applications (digital movies, digital sounds, applets). In this case we will show that a single hash computation will suffice to authenticate the internal blocks. The idea is to embed in the current block a hash of the following block (which in turns includes the hash of the following one and so on...)

The second case is for (potentially infinite) streams which are not known in advance to the signer (for example live feeds, like sports event broadcasting and chat rooms). In this case our solution is less optimal as it requires several hash computations to authenticate a block (although depending on the embedding mechanism these hash computations can be amortized over the length of the block). The size of the embedded authentication information is also an issue in this case. The idea here is to use fast 1-time signature schemes (introduced in $[11,12])$ to authenticate the intemal blocks. So block $i$ will contain a 1-time public key and also the 1 -time signature of itself with respect to the key contained in block $i-1$. This signature authenticates not only the stream block but also the 1-time key attached to it.

\subsection{Related Work}

Some of the ideas involved in the solution for unknown streams have appeared previously, although in different contexts and with different usage.

Mixing "regular" signatures with 1 -time signatures, for the purpose of improving efficiency is discussed in [7]. However in that paper the focus is in making the signing operation of a message $M$ efficient by dividing it in two parts. An off-line part in which the signer signs a 1-time public key with his long-lived secret key even before the messages $M$ is known. Then when $M$ has to be sent the signer computes a 1-time signature of $M$ with the authenticated l-time public key and sends out $M$ tagged with the 1-time public key and the two signatures. Notice that the receiver must compute two signature verifications: one on the long-lived 
key and one on the 1-time key. In our scheme we need to make both signing and verification extremely fast, and indeed in our case each block (except for the first) is signed (and hence verified) only once with a 1-time key.

We also use the idea to of using old keys in order to authenticate new keys. This has appeared in several places but always for long-lived keys. Examples include $[1,15,18]$ where this technique is used to build provably secure signature schemes. We stress that the results in $[1,15,18]$ are mostly of theoretical interest and do not yield practical schemes. Our on-line solution somehow mixes these two ideas in a novel way, by using the chaining technique with 1-time keys, embedding the keys inside the stream flow so that old keys can authenticate at the same time both the new keys and the current stream block.

The chaining technique can also be seen as a weak construction of accumulators as introduced in [2]. An accumulator for $k$ blocks $B_{1}, \ldots, B_{k}$ is a single value $A C C$ that allows a signer to quickly authenticate any of the blocks in any particular order. Accumulators based on the RSA assumption were proposed in [2]. In our case we have a much faster construction based on collision-free hash functions, since we exploit the property that the blocks must be authenticated in a specific order.

\section{Preliminaries}

In the following we denote with $n$ the security parameter. We say that a function $\epsilon(n)$ is negligible if for all $c$, there exists an $n_{0}$ such that, for all $n>n_{0}$, $\epsilon(n)<1 / n^{c}$.

COLLISION-RESISTANT HASH FUNCTIONS. Let $H$ be a function that map arbitrarily long binary strings into elements of a fixed domain $D$. We say that $H$ is a collisionresistant hash function if any polynomial time algorithm who is given as input the values $H\left(x_{i}\right)$ on several adaptively chosen values $x_{i}$, finds a collision, i.e. a pair $(x, y)$ such that $x \neq y$ and $H(x)=H(y)$, only with negligible probability. MD5 [16] and SHA-1 [14] are conjectured collision-resistant hash functions.

SIGNATURE SCHEMES. A signature scheme is a triplet $(G, S, V)$ of probabilistic polynomial-time algorithms satisfying the following properties:

- $G$ is the key generator algorithm. On input $1^{n}$ it outputs a pair $(S K, P K) \in$ $\{0,1\}^{2 n} . S K$ is called the secret (signing) key and $P K$ is called the public (verification) key.

- $S$ is the signing algorithm. On input a message $M$ and the secret key $S K$, it outputs a signature $\sigma$.

$-V$ is the verification algorithm. For every $(P K, S K)=G\left(1^{n}\right)$ and $\sigma=$ $S(S K, M)$, it holds that $V(P K, \sigma, M)=1$. 
In [9] security for signature schemes is defined in several variants. The strongest variant is called "existential unforgeability against adaptively chosen message attack". That is, we require that no efficient algorithm will be able to produce a valid signed message, even after seeing several signed messages of its choice.

STREAM SIGNATURES. We define a stream to be a (possibly infinite) sequence of blocks $\mathcal{B}=B_{1}, B_{2}, \ldots$ where each $B_{i} \in\{0,1\}^{c}$ for some constant ${ }^{3} c$.

We distinguish two cases. In the first case we assume that the stream is finite and known to the sender in advance. We call this the off-line case. Conversely in the on-line case the signer must process one (or a few) block at the time with no knowledge of the future part of the stream.

Definition 1. An off-line stream signature scheme is a triplet $(G, S, V)$ of probabilistic polynomial-time algorithms satisfying the following properties:

- $G$ is the key generator algorithm. On input $1^{n}$ it outputs a pair $(S K, P K) \in$ $\{0,1\}^{2 n} . S K$ is called the secret (signing) key and $P K$ is called the public (verification) key.

- $S$ is the signing algorithm. On input a finite stream $\mathcal{B}=B_{1}, \ldots, B_{k}$ and the secret key $S K$ algorithm $S$ outputs a new stream $B^{\prime}=B_{1}^{\prime}, \ldots, B_{k}^{\prime}$ where $B_{i}^{\prime}=\left(B_{i}, A_{i}\right)$.

$-V$ is the verification algorithm. For every $(P K, S K)=G\left(1^{n}\right)$ and $B^{\prime}=$ $S(S K, \mathcal{B})$, it holds that $V\left(P K, B_{1}^{\prime}, \ldots, B_{i}^{\prime}\right)=1$ for $1 \leq i \leq k$.

Notice that we modeled the off-line property by the fact that the signing algorithm is given the whole stream in advance. Yet the verifier is required to authenticate each prefix of the scheme without needing to see the rest of the stream. As it will become clear in the following our algorithms will not require the off-line verifier to store the whole past stream either.

Definition 2. An on-line stream signature scheme is a triplet $(G, S, V)$ of probabilistic polynomial-time algorithms satisfying the following properties:

- $G$ is the key generator algorithm. On input $1^{n}$ it outputs a pair $(S K, P K) \in$ $\{0,1\}^{2 n} . S K$ is called the secret (signing) key and $P K$ is called the public (verification) key.

$-S$ is the signing algorithm. Given a (possibly infinite) stream $\mathcal{B}=B_{1}, \ldots$, algorithm $S$ with input the secret key $S K$ process each block one at the time, i.e.,

$$
S\left(S K, B_{1}, \ldots, B_{i}\right)=B_{i}^{\prime}=\left(B_{i}, A_{i}\right)
$$

\footnotetext{
The assumption that the blocks have all the same size is not really necessary. We just make it for clarity of presentation.
} 
- $V$ is the verification algorithm. For every $(P K, S K)=G\left(1^{n}\right)$ and $B_{1}^{\prime}, B_{2}^{\prime}, \ldots$ such that $B_{i}^{\prime}=S\left(S K, B_{1}, \ldots, B_{i}\right)$ for all $i$, it holds that $V\left(P K, B_{1}^{\prime}, \ldots, B_{i}^{\prime}\right)=$ 1 for all $i$.

Notice that in the on-line definition we have the signer process each block "on the fly" so knowledge of future blocks is not needed. In this case also the definition seems to requires knowledge of all past blocks for both signer and verifier, however this does not have to be the case (indeed in our solution some past blocks may be discarded).

The above definitions say nothing about security. In order to define security for stream signing we use the same notions of security introduced in [9]. That is, we require that no efficient algorithm will be able to produce a valid signed stream, even after seeing several signed streams. However notice that given our definition of signed streams, a prefix of a valid signed stream is itself a valid signed stream. So the forger can present a "different" signed stream by just taking a prefix of the ones seen before. However this hardly constitutes forgery, so we rule it out in the definition. With $\mathcal{B}^{(1)} \subseteq \mathcal{B}^{(2)}$ we denote the fact that $\mathcal{B}^{(1)}$ is a prefix of $\mathcal{B}^{(2)}$.

Definition 3. We say that an off-line (resp. on-line) stream signature scheme $(G, S, V)$ is secure if any probabilistic polynomial time algorithm $F$, who is given as input the public key $P K$ and adaptively chosen signed streams $\mathcal{B}^{\prime(j)}$, outputs a new previously unseen valid signed stream $\mathcal{B}^{\prime} \nsubseteq \mathcal{B}^{(j)} \forall j$ only with negligible probability.

For signed streams we slightly abuse the notation: when we write $\mathcal{B}^{\prime(1)} \nsubseteq \mathcal{B}^{\prime(2)}$ we mean that not only $\mathcal{B}^{\prime(1)}$ is not a prefix of $\mathcal{B}^{(2)}$ but also the underlying "basic" unsigned streams are in the same relationship, i.e. $\mathcal{B}^{(1)} \nsubseteq \mathcal{B}^{(2)}$.

This is the definition of existential unforgeability against adaptive chosen message attack, the strongest of the notions presented in [9]. Following [9] weaker variants can be defined.

\section{The Off-Line Solution}

In this case we assume that the sender knows the entire stream in advance. (e.g., music/movie broadcast). Assume for simplicity that the stream is such that it is possible to reserve 20 bytes of extra authentication information in a block of size c. 
The stream is logically divided into blocks of size $c$. The receiver has a buffer of size $c$. The receiver first receives the signature on the 20 byte hash (e.g., SHA-1) of the first block. After verification of the signature the receiver knows what the hash of the first block should be and then starts receiving the full stream and starts computing its hash block by block. When the receiver receives the first block, it checks its hash against what the signature was verified upon. If it matches, it plays the block otherwise it rejects it and stops playing the stream. How are other blocks authenticated? The key point is that the first block contains the 20 byte hash of the second block, the second block contains the 20 byte hash of the third block and so on... Thus, after the first signature check, there are just hashes to be checked for every subsequent block.

In more detail, let $(G, S, V)$ be a regular signature scheme. The sender has a pair of secret-public key $(S K, P K)=G\left(1^{n}\right)$ of such signature scheme. Also let $H$ be a collision-resistant cryptographic hash function. If the original stream is

$$
\mathcal{B}=B_{1}, B_{2}, \ldots, B_{k}
$$

and the resulting signed stream is

$$
\mathcal{B}^{\prime}=B_{0}^{\prime}, B_{1}^{\prime}, B_{2}^{\prime}, \ldots, B_{k}^{\prime}
$$

the processing is done backwards on the original stream as follows:

$$
\begin{gathered}
B_{k}^{\prime}=<B_{k}, 00 \ldots 0> \\
B_{i}^{\prime}=<B_{i}, H\left(B_{i+1}^{\prime}\right)>\text { for } i=1, \ldots, k-1 \\
B_{0}^{\prime}=<H\left(B_{1}^{\prime}\right), S\left(S K, H\left(B_{1}^{\prime}\right)\right)>
\end{gathered}
$$

Notice that on the sender side, computing the signature and embedding the hashes requires a single backwards pass on the stream, hence the restriction that the stream is fully known in advance.

The receiver verifies the signed stream as follows: on receiving $B_{0}^{\prime}=\left\langle B, A_{0}\right\rangle$ she checks that

$$
V\left(P K, A_{0}, B\right)=1
$$

then on receiving $B_{i}^{\prime}=<B_{i}, A_{i}>$ (for $i \geq 1$ ) the receiver accepts $B_{i}$ if

$$
H\left(B_{i}^{\prime}\right)=A_{i-1}
$$

Thus the receiver has to compute a single public-key operation at the beginning, and then only one hash evaluation per block. Notice that no big table is needed in memory. 


\section{The On-Line Solution}

In this case the sender does not know the entire stream in advance (e.g, live broadcast). In this scenario it is important that also the operation of signing (and not just verification) be fast, since the sender himself is bound to produce an authenticated stream at a potentially high rate.

ONE-TIME SIGNATURES. In the following we will use a special kind of signature scheme introduced in $[11,12]$. These are signatures which are much faster to compute and verify than regular signatures since they are based on one-way functions and do not require a trapdoor function. Conjectured known one-way functions (as DES or SHA-1) are much more efficient then the known conjectured trapdoor functions as RSA. However these schemes cannot be used to sign an arbitrary number of messages but only a prefixed number of them (usually one). Several other 1-time schemes have been proposed $[7,3,4]$; in Section 6 we discuss possible instantiations for our purpose.

In this case also the stream is split into blocks. Initially the sender sends a signed public key for a 1-time signature scheme. Then he sends the first block along with a 1-time signature on its hash based on the 1-time public key sent in the previous block. The first block also contains a new 1-time public key to be used to verify the signature on the 2nd block and this structure is repeated in all the blocks.

More in detail: let us denote with $(G, S, V)$ a regular signature scheme and with $(g, s, v)$ a 1-time signature scheme. With $H$ we still denote a collision-resistant hash function. The sender has long-lived keys $(S K, P K)=G\left(1^{n}\right)$. Let

$$
\mathcal{B}=B_{1}, B_{2}, \ldots
$$

be the original stream (notice that in this case we are not assuming the stream to be finite) and

$$
\mathcal{B}^{\prime}=B_{0}^{\prime}, B_{1}^{\prime}, B_{2}^{\prime}, \ldots
$$

the signed stream constructed as follows. For each $i \geq 1$ let us denote with $\left(s k_{i}, p k_{i}\right)=g\left(1^{n}\right)$ the output of an independent run of algorithm $g$. Then

$$
B_{0}^{\prime}=\left\langle p k_{0}, S\left(S K, p k_{0}\right)>\right.
$$

(public keys of 1-time signature schemes are usually short so they need not to be hashed before signing)

$$
B_{i}^{\prime}=<B_{i}, p k_{i}, s\left(s k_{i-1}, H\left(B_{i}, p k_{i}\right)\right)>\text { for } i \geq 1
$$


Notice that apart from a regular signature on the first block, all the following signatures are 1-time ones, thus much faster to compute (including the key generation, which however does not have to be done on the fly.)

The receiver verifies the signed stream as follows. On receiving $B_{0}^{\prime}=\left\langle p k_{0}, A_{0}\right\rangle$ she checks that

$$
V\left(P K, A_{0}, p k_{0}\right)=1
$$

and then on receiving $B_{i}^{\prime}=<B_{i}, p k_{i+1}, A_{i}>$ she checks that

$$
v\left(p k_{i-1}, A_{i}, H\left(B_{i}, p k_{i}\right)\right)=1
$$

whenever one of these checks fails, the receiver stops playing the stream. Thus the receiver has to compute a single public-key operation at the beginning, and then only one 1-time signature verification per block.

\section{Proofs of Security}

We were able to prove the security of our stream signature schemes according to the definitions presented in Section 2, provided that the underlying components used to build the schemes are secure on their own. The proofs of the following theorems appear in the full version of the paper [8] due to space limitations.

THE OFF-LINE CASE. Let us denote with $\left(\mathcal{G}_{\circ f f}, \mathcal{S}_{\text {off } f}, \mathcal{V}_{\text {of } f}\right)$ the off-line stream signature scheme described in Section 3. With $(G, S, V)$ let us denote the "regular" signature scheme and with $B$ the hash function used in the construction. The following holds.

Theorem 4. If $(G, S, V)$ is a secure signature scheme and $H$ is a collisionresistant hash function then the resulting stream signature scheme $\left(\mathcal{G}_{\text {of } f}, \mathcal{S}_{\text {off } f}, \mathcal{V}_{\text {off }}\right.$ is secure.

THE ON-LINE CASE. Let us denote with $\left(\mathcal{G}_{o n}, \mathcal{S}_{\text {on }}, \mathcal{V}_{\text {on }}\right)$ the on-line stream signature scheme described in Section 4. With $(G, S, V)$ let us denote the "regular" signature scheme, with $(g, s, v)$ the one-time signature scheme and with $H$ the hash function used in the construction. The following holds.

Theorem 5. If $(G, S, V)$ and $(g, s, v)$ are secure signature schemes and $H$ is a collision-resistant hash function then the resulting stream signature scheme $\left(\mathcal{G}_{\text {on }}, \mathcal{S}_{\text {on }}, \mathcal{V}_{\text {on }}\right)$ is secure. 
Remark 1: In the bodies and proofs of the above theorems we meant security as "existential unforgeability against adaptively chosen message attack". However the theorems hold for any notion of security defined in [9], that is the stream signature scheme inherits the same kind of security of the underlying signature scheme(s) provided that the hash function is collision-resistant.

Remark 2: The statements of the above theorems are valid not only in asymptotic terms, but have also a concrete interpretation which ultimately is reflected in the key lengths used in the various components in order to achieve the desired level of security of the full construction. It is not hard to see, by a close analysis of the proofs, that the results are pretty tight. That is, a forger for the stream signing scheme can be transformed into an attacker for one of the components (the hash function, the regular signature scheme and, a little less optimally, the 1-time signature scheme) which runs in about the same time, asks the same number of queries and has almost the same success probability. This is tums means that there is no major degradation in the level of security of the compound scheme and thus the basic components can be run with keys of ordinary length.

\section{Implementation Issues}

\subsection{The Choice of the One-Time Signature Scheme}

Several one-time schemes have been presented in the literature, see for example $[11,12,7,3,4]$. The main parameters of these schemes are signature length and verification time. In the solutions we know, these parameters impose conflicting requirements, i.e. if one wants a scheme with short signatures, verification time goes up, while schemes with longer signatures can have a much shorter verification time. In our on-line solution we would like to keep both parameters down. Indeed the verification should be fast enough to allow the receiver to consume the stream blocks at the same input rate she receives them. At the same time, since the signatures are embedded in the stream, it's important to keep them small so that they will not reduce the throughput rate of the original stream.

We present a scheme which obtain a reasonable compromise. In the final paper we will present several more schemes. The scheme is based on a 1-way function $F$ in a domain $D$. It also uses a collision resistant hash function $H$. The scheme allows signing of a single $m$-bit message. It is based on a combinations of ideas from $[11,19]$. Here are the details of the scheme.

Key Generation. Choose $m+\log m$ elements in $D$, let them be $a_{1}, \ldots, a_{m+\log m}$. This is the secret key. The publick key is

$$
p k=H\left(F\left(a_{1}\right), \ldots, F\left(a_{m+\log m}\right)\right)
$$


Signing Algorithm. Let $M$ be the message to be signed. Append to $M$ the binary representation of the number of zero's in $M$ 's binary representation. Call $M^{\prime}$ the resulting binary string. The signature of $M$ is $s_{1}, \ldots, s_{m+\log m}$ where $s_{i}=a_{i}$ if the $i^{\text {th }}$ bit of $M^{\prime}$ is 1 otherwise $s_{i}=F\left(a_{i}\right)$.

Verification Algorithm. Check if

$$
H\left(t_{1}, \ldots, t_{m+\log m}\right)=p k
$$

where $t_{i}=s_{i}$ if $i^{\text {th }}$ bit of $M^{\prime}$ is 0 otherwise $t_{i}=F\left(s_{i}\right)$.

Security. Intuitively this scheme is secure since it is not possible to change a 0 into a 1 in the binary representation of the message $M$ without having to invert the function $F$. It is possible to change a 1 into a 0 , but that will increase the number of 0 's in the binary representation of $M$ causing a bit to flip from 0 to 1 in the last $\log m$ bits of $M^{\prime}$, and so forcing the attacker to invert $F$ anyway.

Parameters. This scheme has signature length $|D|(m+\log m)$ where $|D|$ is the number of bits required to represent elements of $D$. The receiver has to compute 1 hash computation of $H$ plus on the average $\frac{m+\log m}{2}$ computations of $F$.

In practice we assume that $F$ maps 64-bit long strings into 64-bit long strings. Since collision resistance is not required from $F$ we believe this parameter is sufficient. Conjectured good $F$ 's can be easily constructed from efficient block ciphers like DES or from fast hash function like MD5 or SHA-1. ${ }^{4}$ Similarly $H$ can be instantiated to MD5 or SHA-1. In general we may assume $m$ to be 128 or 160 if the message to be signed if first hashed using MD5 or SHA-1.

The SHA-1 implementation bas then signatures which are 1344 bytes long. The receiver has to compute $F$ around 84 times on the average. With MD5 the numbers become 1080 bytes and 68 respectively. When used in our off-line scheme one also has to add 16 bytes for the embedding of the public key in the stream.

Remark: Comparing the RSA signature scheme with verification exponent 3 with the above schemes, one could wonder if the verification algorithm is really more efficient ( 2 multiplications verses 84 hash computations). Typical estimates today are that an RSA verification is comparable to 100 hash computations. However we remind the reader that we are trying to improve both signature generation and verification as this scheme is used in the on-line case and as such both operations have to be performed on-line and thus efficiently. The improvement in signature generation is much more substantial.

\footnotetext{
4 As a cautionary remark to prevent attacks where the attacker builds a large table of evaluations of $F$, in practice $F$ could be made different for each signed stream (or for each large portion of the signed stream) by defining $F(x)$ to be $G(S a l t \| X)$ where $G$ is a one-way 128 bit to 64 bit function, and the $S a l t$ is generated at random by the signer once for each stream or large pieces thereof.
} 


\subsection{Non-Repudiation}

In case of a legal dispute over a content of a signed stream the receiver must bring to court some evidence. If the receiver saves the whole stream, then there is no problem. However in some cases, for example because of memory limitations, the receiver may be forced to discard the stream data after having consumed it. In these cases what should she save to protect herself in case of a legal dispute?

In the off-line solution, assuming the last block of the signed stream always has a special reserved value for the hash-chaining field, (say all 0's) she needs to save only the first signed block. Indeed this proves that she received something from the sender. Now we could conceivably move the burden of proof to the sender to reconstruct the whole stream that matches that first block and ends with the last block which has the reserved value for the hash-chaining field.

Similarly in the on-line solution, at a minimum the receiver needs to save the first signed block and all 1-time signatures and have the sender reconstruct the stream. However in practice, this may still be too much to save. In the final paper we will discuss various modifications to the on-line scheme that can be used in practice to substantially reduce the data that the receiver needs to store.

\subsection{Hybrid Schemes}

In the on-line scheme, the length of the embedded authentication information is of concern as it could cut into the throughput of the stream. In order to reduce it hybrid schemes can be considered. In this case we assume that some asynchrony between the sender and receiver is acceptable.

Suppose the sender can process a group (say 20) of stream blocks at a time before sending them. With a pipelined process this would only add an initial delay before the stream gets transmitted. The sender will sign with a one-time key only 1 block out of 20 . The 20 blocks in between these two signed blocks will be authenticated using the off-line scheme. This way the long 1-time signatures and the the verification time can be amortized over the 20 blocks.

A useful feature of our proposed 1-time signature scheme is that it allows the verification of (the hash of) a message bit by bit. This allows us to actually "spread out" the signature bits and the verification time among the 20 blocks. Indeed if we assume that the receiver is allowed to play at most 20 blocks of unauthenticated information before stopping if tampering is detected we can do the following. We can distribute the signature bits among the 20 blocks and verify the hash of the first block bit by bit as the signature bits arrive. This maintains the stream rate stable since we do not have long signatures sent in a single block and verification now takes 3-4 hash computations per block, on every block. 
It is also possible to remove the constraint on playing 20 blocks of unauthenticated information before tampering is detected. This requires a simple modification to our on-line scheme. Instead of embedding in block $B_{i}$ its own 1-time signature, we embed the signature of the next block $B_{i+1}$. This means that in the on-line case blocks have to be processed two at a time now. When this modification is applied to the hybrid scheme, the signature bits in the current 20 blocks are used to authenticate the following 20 blocks so unauthenticated information is never played. However this means that now the sender has to process 40 blocks at a time in the hybrid scheme.

\section{Applications}

MPEG VIDEO AND AUDIO. In the case of MPEG video and audio, there are several methods for embedding authentication data. Firstly, the Video Elementary stream has a USER-DATA section where arbitrary user defined information can be placed. Secondly, the MPEG system layer allows for an elementary data stream to be multiplexed synchronously with the packetized audio and video streams. One such elementary stream could carry the authentication information. Thirdly, techniques borrowed from digital watermarking can be used to embed information in the audio and video itself at the cost of slight quality degradation. In the case of MPEG video since each frame is fairly large, (hundreds of kilobits) and the receiver is required to have a buffer of at least $1.8 \mathrm{Mbits}$, both the off-line as well as the on-line solutions can be deployed without compromising picture quality. In the case of audio however, in the extreme case the bit rate could be very low (e.g., 32Kbits/s) and each audio frame could be small (approx. 1000 bytes) and the receiver's audio buffer may be tiny ( $<2$ Kbytes). In such extreme cases the on-line method, which requires around 1000 bytes of authentication information per block cannot be used without seriously cutting into audio quality. For these extreme cases, the best on-line strategy would be either to send the authentication information via a separate but multiplexed MPEG data stream. For regular MPEG audio, if the receiver has a reasonably sized buffer (say $32 \mathrm{~K}$ ) then by having a large audio block (say $20 \mathrm{~K}$ ) our on-line scheme would a server-introduced delay of approximately 5-6 seconds and a $5 \%$ quality degradation. If the receiver buffer is small but not tiny (say $3 \mathrm{~K}$ ) a hybrid scheme would work: as an example of a scheme that can be built one could use groups of 33 hash-chained blocks of length 1000 bytes each; this would typically result in a $5 \%$ degradation and a server initial delay in the 20 second range.

JAVA. In the original version of java (JDK 1.0), for an applet coming from the network, first the startup class was loaded and then additional classes were (down) loaded by the class loader in a lazy fashion as and when the running applet first 
attempted to access them. Since our ideas apply not only to streams which are a linear sequence of blocks but in general to trees as well (where one block can invoke any of its children), based on our model, one way to sign java applets would be to sign the startup class and each downloaded class would have embedded in it the hashes of the additional classes that it downloads directly. However for code signing, Javasofthas adopted the multiple signature and hash table based approach in JDK 1.1, where each applet is composed of one or several Java archives, each of which contains a signed table of hashes (the manifest) of its components. It is our belief that once java applets become really large and complex the shortcomings of this approach will become apparent: (1) the large size of the hash table in relation to the classes actually invoked during a run. This table has to fully extracted and authenticated before any class gets authenticated; (2) the computational cost of signing each of the manifests if an applet is composed of several archives; (3) accommodating classes or data resources which are generated on the fly by the application server based on a client request.

These could be addressed by using some of our techniques. Also the problem of how to sign audio/video streams will have to be considered in the future evolution of Java, since putting the hash of a large audio/video file in the manifest would not be acceptable.

BROADCAST APPLICATIONS. Our schemes (both the off-line and the on-line one) can be easily modified to fit in a broadcast scenario. Assume that the stream is being sent to a broadcast channel with multiple receivers who dynamically join or leave the channel. In this case a receiver who joins when the transmission is already started will not be able to authenticate the stream since she missed the first block that contained the signature. Both schemes however can be modified so that every once in a while apart from the regular chaining information, there will also be a regular digital signature on a block embedded in the stream. Receivers who are already verifying the stream via the chaining mechanism can ignore this signature whereas receivers tuned in at various time will rely on the first such signature they encounter to start their authentication chain. A different method to authenticate broadcasted streams, with weaker non-repudiation properties than ours, was proposed in [10].

LONG FILES WHEN COMMUNICATION IS AT COST. Our solution can be used also to authenticate long files in a way to reduce communication cost in case of tampering. Suppose that a receiver is downloading a long file from the Web. There is no "stream requirement" to consume the file as it is downloaded, so the receiver could easily receive the whole file and then check a signature at the end. However if the file has been tampered with, the user will be able to detect this fact only at the end. Since communication is at a cost (time spent online, bandwidth 
wasted etc) this is not a satisfactory solution. Using our solution the receiver can interrupt the transmission as soon as tampering is detected thus saving precious communication resources.

SIGNATURE BASED CONTENT-FILTERING AT PROXIES. Recently there has been interest in using digital signatures as a possible way to filter content admitted in by proxy servers through firewalls. Essentially when there is a firewall and one wishes to connect to an external server, then this connection can only be done via a proxy server. In essence one establishes a connection to a proxy and the proxy establishes a separate connection to the external server (if that is permitted). The proxy then simulates a connection between the internal machine and the external machine by copying data between the two connections. There has been some interest in modifying proxies so that they would only allow signed data to flow from the external server to the internal machine. However, since the proxy is only copying data as it arrives from the external connection into the internal connection and it cannot store all the incoming data before transferring it, the proxy cannot use a regular signature scheme for solving this problem. However, it is easy to see that in the proxy's view the data is a stream. Hence if there could be some standardized way to embed authentication data in such streams then techniques from this paper would prove useful in solving this problem.

\section{Acknowledgments}

We would like to thank Hugo Krawczyk for his advice, guidance and encouragement for this work. We would also like to thank Ran Canetti and Mike Wiener for helpful discussions.

\section{References}

1. M. Bellare, S. Micali. How to Sign Given any Trapdoor Permutation. J. of the $A C M, 39(1): 214-233,1992$.

2. J. Benaloh, M. de Mare. One-Way Accumulators: A Decentralized Alternative to Digital Signatures. Advances in Cryptology-EUROCRYPT'93. LNCS, vol.765, pp.274-285, Springer-Verlag, 1994.

3. D. Bleichenbacher, U. Maurer. Optimal Tree-Based One-time Digital Signature Schemes. STACS'96, LNCS, Vol. 1046, pp.363-374, Springer-Verlag. 
4. D. Bleichenbacher, U. Maurer. On the efficiency of one-time digital signatures. Advances in Cryptology-ASYACRYPT'96, to appear.

5. W. Diffie, M. Hellman. New Directions in Cryptography. IEEE Transactions on Information Theory, IT-22(6):74-84, 1976.

6. T. ElGamal. A Public-Key Cryptosystem and a Signature Scheme based on Discrete Logarithms. IEEE Transactions on Information Theory, IT31(4):469-472, 1985.

7. S. Even, O. Goldreich, S. Micali. On-Line/Off-Line Digital Signatures. J. of Cryptology, 9(1):35-67, 1996.

8. R. Gennaro, P. Rohatgi. How to Sign Digital Streams.

Final version available from

http://www.research.ibm.com/security/papers1997.html

9. S. Goldwasser, S. Micali, R. Rivest. A Digital Signature Scheme Secure Against Adaptive Chosen Message Attack. SIAM J. Comp. 17(2):281-308, 1988.

10. G. Itkis. Asymmetric MACs. Rump talk at Crypto'96.

11. L. Lamport. Constructing Digital Signatures from a One-Way Function. Technical Report SRI Intl. CSL 98, 1979.

12. R. Merkle. A Digital Signature based on a Conventional Encryption Function. Advances in Cryptology-Crypto'87. LNCS, vol.293, pp. 369-378, SpringerVerlag, 1988.

13. R. Merkle. A Certified Digital Signature. Advances in Cryptology Crypto'89. LNCS, vol.435, pp. 218-238, Springer-Verlag, 1990.

14. National Institute of Standard and Technology. Secure Hash Standard. NIST FIPS Pub 180-1, 1995.

15. M. Naor, M. Yung. Universal One-Way Hash Functions and their Cryptographic Applications. Proceedings of STOC 1989, pp.33-43.

16. R. Rivest. The MD5 Message Digest Algorithm. Internet Request for Comments. April 1992.

17. R. Rivest, A. Shamir, L. Adleman. A Method for Obtaining Digital Signatures and Public Key Cryptosystems. Comm. of the ACM, 21(2):120-126, 1978.

18. J. Rompel. One-Way Functions are Necessary and Sufficient for Secure Signatures. Proceedings of STOC 1990, pp.387-394.

19. Winternitz. Personal communication to R. Merkle. 